\title{
Piani di fortificazione in Calabria Ultra tra XVI e XVII secolo
}

Fortification plans in Calabria Ultra between the sixteenth and seventeenth centuries

\author{
Francesca Martorano \\ Dipartimento Patrimonio Architettura Urbanistica - Università degli Studi di Reggio Calabria, Reggio Calabria, Italy \\ fmartorano@unirc.it
}

\begin{abstract}
The Crown of Spain acquired, as it is known, the kingdom of Naples in 1504, which as Viceregno it will be part of the Spanish empire for more than two centuries. The empire between the sixteenth and seventeenth centuries was faced with various challenges, both internal and boundary, particularly maritime, attacked by the Ottoman expansion. Urban and coastal fortification plans were prepared and put in place to defend the territory. Calabria was also a participant in this effort, which the Viceroys directed and followed from Naples, with the approval of Madrid. This study examines the projects and achievements implemented in the chronological period under consideration in Calabria Ultra, current provinces of Reggio Calabria, Vibo Valentia, Catanzaro and Crotone. Particular attention is paid to coastal defense plans with the design of new towers, which combined with city walls and/or castles in state-owned or feudal cities, complement the defensive projects of the coasts. It is important to highlight how the types adopted are consistent with coherent implementations implemented in the extended territory of the empire, thus declaring widespread knowledge and cultural identity in the Europe of the time.
\end{abstract}

Keywords: Calabria Ultra, coastal towers, fortification plans.

\section{Premessa}

Per l'esteso profilo costiero che la caratterizza, la Calabria, protesa tra Oriente e Occidente, fu bersaglio sin dalla prima metà del Cinquecento $\mathrm{e}$ per quasi tre secoli di incursioni turchesche e attacchi barbareschi. Studi dettagliati hanno ricostruito la sequenza delle disposizioni emanate a partire dal 1532, quando il cardinale Colonna ideò la fortificazione dei punti strategici della costa, seguito tre anni dopo dal viceré don Pietro da Toledo con molteplici ordinamenti (Hernando Sánchez, 2001, pp. 387-388; Galasso, 2005, p. 483). Infine il successivo viceré, Parafan de Ribera duca di Alcalà, attuò iniziative più efficaci indirizzate a potenziare i nodi nevralgici. Pertanto ben note sono le modalità di progettazione e di realizzazione della rete difensiva costiera del Regno di Napoli e le procedure per il suo mantenimento in efficienza.

La costruzione di torri iniziò in Calabria nel 1564, un anno dopo la prima ordinanza relativa al Principato Citra, cioè al litorale tra Salerno e Agropoli. Occorreva costruirle in vista l'una dell'altra, in modo da costituire una continua e ininterrotta catena di fortificazioni. Il progetto tuttavia procedette lentamente perché gravava eccessivamente sulla popolazione: le università ubicate entro dodici miglia dalla costa dovevano versare 22 grana a fuoco, mentre quelle più lontane dovevano contribuire per la metà. Frequenti furono pertanto le sospensioni dei lavori già avviati (Mafrici, 2009, pp. 742-743). 
Ciò nonostante ai primi del Seicento era avvenuta la realizzazione di torri per l'avvistamento e la segnalazione di eventuali sbarchi del Turco o del barbaresco, come attestato da una relazione non autografa di un Viceré di Napoli (García García, 1993, pp. 69-70).

\section{Il progetto di fortificazione}

La relazione non chiarisce il numero complessivo delle fortificazioni effettivamente costruite e solo di recente la scoperta con i primi studi sul Codice Romano Carratelli ha consentito di presentare alcune valutazioni e di operare un confronto con quanto già noto (Mafrici, 2015; Martorano, 2015). L'inportanza del manoscritto è stata successivamente ribadita (Bianchi, Saeli, 2016) e approfondita su temi specifici (Corrado, 2016; Conti, Macrì, 2016).

Il Codice è composto da 99 fogli privi di numerazione antica, illustrati ad acquerello con prospettive di torri. I disegni occupano mezza pagina, mentre la restante metà è riservata al testo descrittivo che in nove casi prosegue nel verso. La sequenza delle torri è interrotta ad intervalli da castelli e rappresentazioni del territorio con vedute di città ed edifici significativi. Dalle modalità di rappresentazione traspare la procedura seguita nella visita per scegliere i luoghi adatti da fortificare e individuare quali fossero i punti sguarniti su cui occorreva intervenire.

L'ispezione dovette tener conto sia della viabilità esistente che della necessità di restituire efficacemente lo stato dei luoghi, dimostrando come le torri avrebbero potuto assolvere il loro compito. La Visita inizia da Reggio, dal litorale tirrenico in cui la costa è prevalentemente rocciosa e difficilmente accessibile, pertanto ritengo sia stato inevitabile ricorrere all'uso di imbarcazioni. Le torri sono infatti tutte, tranne un'unica eccezione La petra della galera - s. Arena (Fig. 1), raffigurate dal mare, mentre sul versante ionico sabbioso e pianeggiante il punto di vista è generalmente da terra, tranne il tratto costiero tra $\mathrm{Ca}$ po Spartivento e Melito.

Sono classificate accanto alla raffigurazione con un numero e con il nome. Tuttavia questo non sempre è presente e in alcuni casi non vi è identità tra il nome che affianca il disegno e quello contenuto nel testo. Sono apportate anche correzioni nella numerazione progressiva a seguire dalla torre n. 26 la petra della galera - s. Arena e sino alla fine del versante tirrenico della Calabria Ultra, di cui l'ultima torre è quella del Suvero.

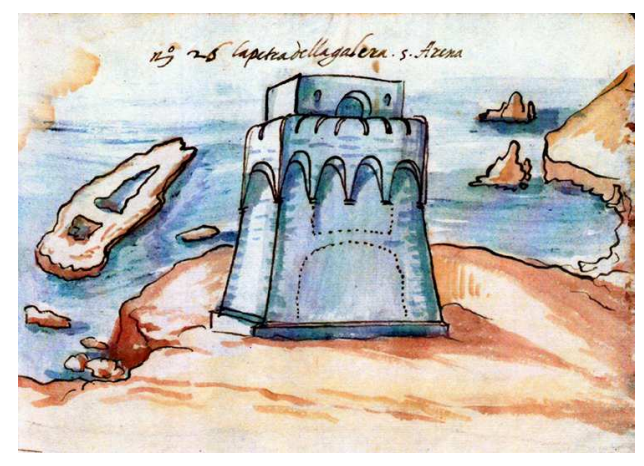

Fig. 1. Torre n. 26 La petra della galera - s. Arena (Codice f. 28).

L'ispezione riprende sullo Ionio dal fiume Neto e nella numerazione di questo litorale esiste una sola correzione: si tratta della Torre di Stalattì, troncoconica, il cui numero 56 è corretto in 57 (Fig. 2). Probabilmente la composizione del volume seguì tempi diversi: dapprima si delineò l'immagine, in un secondo tempo fu scritto il testo che richiese talora la necessità di rettifiche.

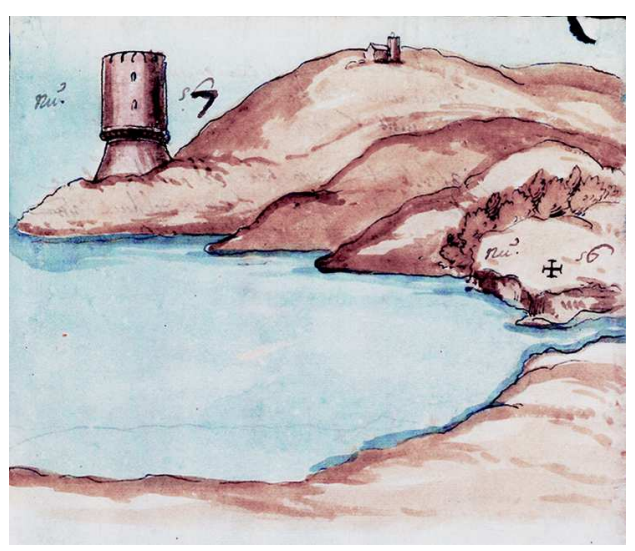

Fig. 2. Torre n. 56 de Stalettì (Codice f. 62).

Anche la qualità degli acquerelli non è omogenea e forse è indizio dell'opera di più artisti. Ho classificato nelle tabelle a fine contributo, accanto al nome, la tipologia e le varianti delle 
torri, sia quelle esistenti che quelle da realizzare o la cui costruzione era stata interrotta. Le torri esistenti a pianta circolare erano in tutto quarantasette, ventuno sul Tirreno e ventisei sullo Ionio, di cui le quattro più antiche cilindriche: torre s. Maria di Tropea (Fig. 3), torre S. Antonio, torre de Jerace detta Paliapoli, torre Petra Teodosa; le restanti circolari con scarpa.

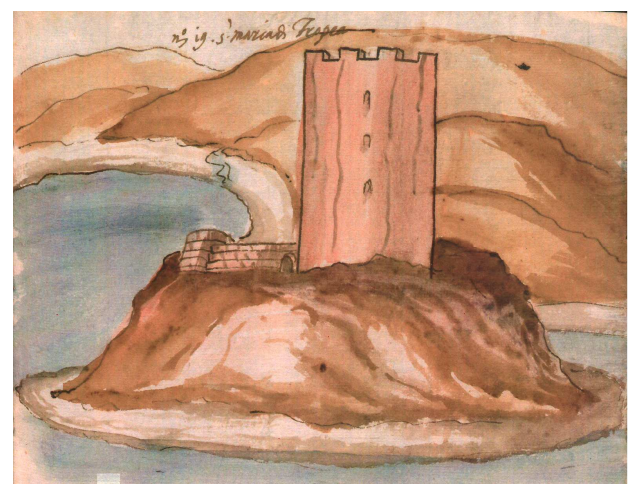

Fig. 3. Torre n. 19 s. Maria di Tropea (Codice f. 20).

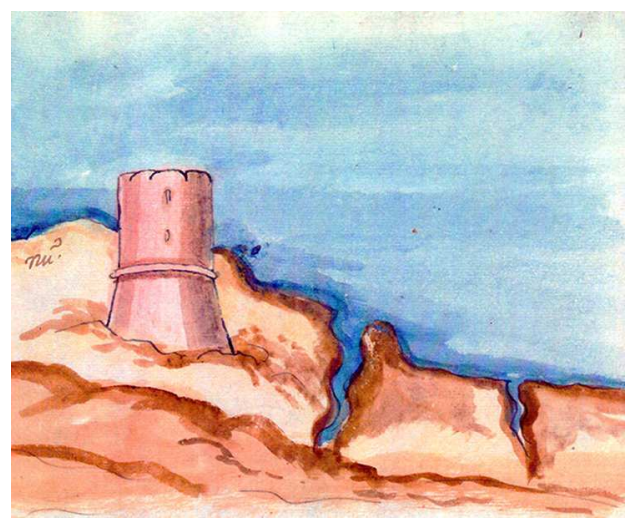

Fig. 4. Torre n. 70 Ciamillarj (Codice f. 73).

L'analisi dei testi mi ha consentito di valutare come le dimensioni fossero diverse in ciascuna struttura, anche se tali varianti sono ignorate nella restituzione grafica. Fanno eccezione un gruppo di cinque torri poste sul litorale ionico: le torri del castellone / de monasterace, Vedera, Casamona, San Fili e Ciamillarj (Fig. 4), che avevano identiche dimensioni sia alla base che nel corpo. Credo che ciò non sia casuale ma risponda ad un progetto unitario di fortificazione, sinora mai messo in risalto.
Le torri non completate al momento della Visita erano otto, di cui sette a pianta quadrangolare scarpata e una circolare, mentre quelle da edificare ex novo erano in tutto cinquantacinque, di cui per quarantasette si disegna la volumetria.

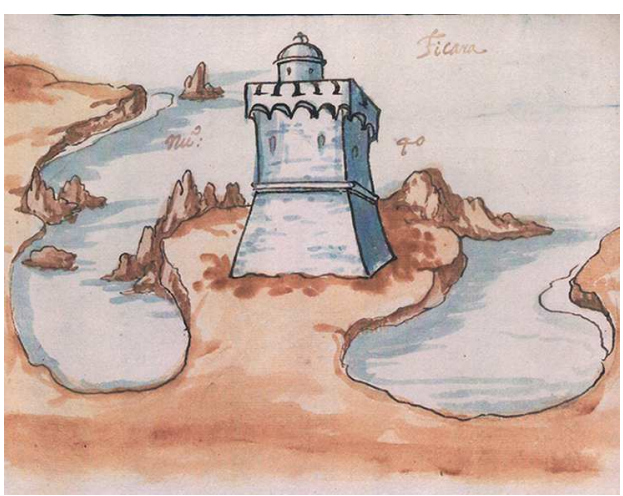

Fig. 5. Torre n. 40 Ficara (Codice f. 46).

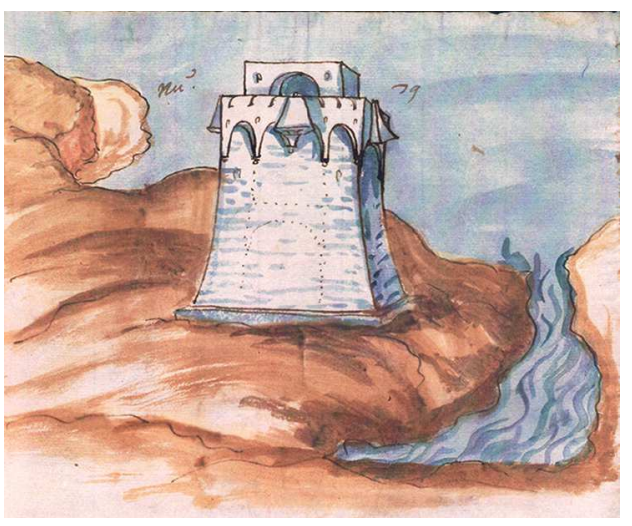

Fig. 6. Torre n. 79 in luoco detto Serlione (Codice f. 83).

Sono raggruppabili in due tipologie, con piccole varianti legate alla presenza di elementi secondari: le bertesche, i vani sul terrazzo per il deposito delle munizioni, le garitte angolari. Una tipologia (Q1) ha la base a scarpa, con il redondone che segna lo stacco con il secondo livello parallelepipedo concluso da beccatelli e archetti (Fig. 5), l'altra corrisponde alla cosiddetta torre "vicereale", tronco-piramidale con troniere in controscarpa, che ho identificato come Q2 (Fig. 6). Sia l'uno che l'altro tipo sono proposte in situazioni territoriali diverse e il manoscritto non contiene alcuna indicazione che indirizzi la scelta a favore dell'uno o dell'altro, dimostrando così che 
ambedue tipi erano ritenuti idonei a svolgere efficacemente la difesa (Martorano, 2015, p. 77).

In relazione alla dislocazione delle torri il Codice conferma che la costruzione delle nuove era consigliata per tutelare i luoghi con presenza d'acqua e la cui conformazione avrebbe potuto consentire facilmente agli infedeli l'ormeggio e lo sbarco. Anche l'eminenza dei luoghi era un criterio dirimente perché permetteva di segnalare il nemico. Lo si esplicita per la torre di petra teodosa (Fig. 7), che viene ritenuta non valida perché priva di contatto visivo con quella di porto palizzi. Si proponeva pertanto di sostituirla e per il promontorio scelto si afferma: "luoco molto atto et buono a scovrire tutti e doi torri".

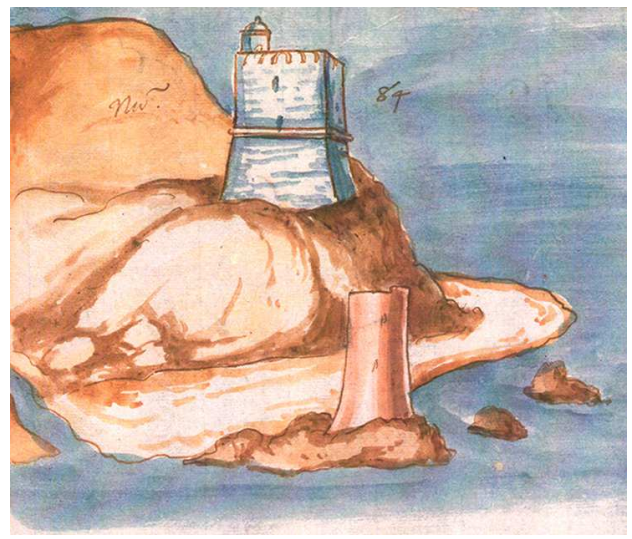

Fig. 7. Torre n. 84 petra teodosa (Codice f. 88).

Il manoscritto dimostra anche attenzione verso aree con presenza di attività economiche: saline, stalle regie, mulini, tonnare, presso le quali suggerisce l'edificazione di nuove torri.

Per quanto riguarda l'intervallo da adottare tra una torre e l'altra, tale misura è espressa solo per il tratto di litorale tra Bagnara e Palmi, dove è fissata in due miglia, mentre per la vicina Sicilia lo Spannocchi stabilisce la distanza modulare di tre (Mazzamuto, 1986, p. 19).

Il piano difensivo del litorale era integrato da castelli e città fortificate. Il Codice registra la presenza degli uni: La Rocchetta di Briatico, il castello di Bivona, Le Castella, Reggio, e delle altre, e per otto città, sette costiere: Scilla, Bagnara, Tropea, Pizzo, Crotone, terra delle Castella, Roccella di Castelvetere, e una sola nell'entroterra, Gerace, dedica una pagina intera alla raffigurazione, con il testo descrittivo assente. In altri casi le raffigurazioni dei centri fortificati sono inserite in una veduta territoriale, pertanto più ridotte nelle dimensioni e sintetiche, ma per tutte è posta attenzione agli elementi difensivi, colorati in rosa se esistenti o in azzurro se di progetto. Questa diversa cromia è utilizzata anche nella raffigurazione delle torri.

\section{L'attuazione del progetto nella metà del XVII secolo}

Per il Codice, sulla base di indicazioni contenute nel testo, ho proposto la datazione della Visita agli anni post 1594-1597, mentre la stesura è successiva (Martorano, 2015, pp. 87-88).

Mi sembra pertanto interessante porre a confronto i suoi dati, la sua proposta progettuale e l'identificazione tipologica delle torri con l'elenco ricavato dalla Carta manoscritta di Stigliola che Vladimiro Valerio data al 1595 (Valerio, 2015, pp. 126-131) e quello contenuto nella relazione che nel 1638 il vicario Giovan Tomaso Blanch inviò al vicerè duca di Medina (Valente, 1964, pp. 620-624). Sia Stigliola che Blanch fanno riferimento a torri esistenti e pertanto ho effettuato il riscontro con il Codice e accertato quanto fosse stato realizzato (Tabb. 1-3). Le torri cilindriche, le circolari con scarpa e le poche quadrangolari già costruite o realizzate solo in parte sono tutte presenti anche in Stigliola.

Va precisato che in due casi, la torre n. 4 Lo cavallo e la torre $\mathrm{n} .84$ petra teodosa, la sequenza della Carta Stigliola è diversa da quella elencata dal Codice per una inversione dei nomi, in pochi altri cambia la denominazione pur trattandosi dello stesso edificio, come ad esempio sul Tirreno la torre in luogo di cannameli territorio di Fiumara di Muro indicata in Stigliola come torre flumaro de muro.

Quattro torri sul versante ionico: le nn. 46-49 penso possano corrispondere alle torri delle $\mathrm{Ca}$ stelle, de iacopani, lacozzia, de tacina. L'identificazione che propongo deriva dalle località in cui si sarebbero dovute costruire la prima e l'ultima. La n. 46 "in luoco detto la scala dele Castelle" e la n. 49 "in luoco detto Bocca del fiume di tacina". Va precisato tuttavia che in 
Blanch la torre de li Castelle è senza dubbio quella del castello.

Solo per pochissime altre, due o tre al massimo, come evidenziato nelle tabelle 1 e 2 , non vi è riscontro, perché gli altri vuoti sono spiegabili con lo scambio di sequenza o la duplicazione del nome. Nel Codice due sole strutture hanno doppia denominazione: la n. 30 Torre Santo Petro / santa Venera, in Stigliola S. Venere, e la n. 73 torre di Jerace detta Paliapoli che in Stigliola è indicata come due differenti strutture.

Nelle Tabelle, nell'elenco tratto dal Codice, ho utilizzato il carattere tondo per il nome che affianca il disegno e il corsivo per il nome tratto dal testo; in Stigliola e in Blanch il corsivo per i nomi dei pochi casi in cui le torri hanno denominazione diversa dal Codice anche se, per affinità nei dati topografici, ritengo si tratti delle
N. CODICE Romano Carratelli post 1594-1597

Torre La catona / Il Palazzo

Torre, in luoco di cannameli

3 Torre La coda della volpe / lo Pirayno

4 Torre Lo cavallo

5 Torre Porto s. Grigorio

6 Torre La tonnara serra et mulini

7 Torre di capo di rochi

8 Torre san bastiano

9 Torre santo Leo

10 Torre, a doi miglia da Santo Leo

11 Torre di Palmi o S. Francesco

12 Torre La tonnara

13 Torre Le petri nere

14 GIOYA et torre

Torre da costruire

Torre di Rosarno

Torre da costruire, verso il fiume ditto il carosello Torre da costruire, alla marina di Nicotera

Torre di Nicotera - s. Maria delli agnone

Torre, da costruire in territorio di Curcurina

Torre s. maria di Tropea / s. Maria di Loreto

Torre batticano / de capo de vaticano

Torre laruffa

Torre santa dominica

Torre tonnara e acqua di praialia

Torre Zambrone + torre da costruire

Torre in La Gorna di Cocone

Torre La petra della galera / s. Arena

Torre dell'Imperatore

OCHETTA DE BRIATICO

Torre san nicola del porto

Torre la cala dili schiabbachi

\section{Torre Santo Petro / santa Venera}

Torre Langitula et fiume + torre da costruire

Torre di mezza praya

torre dell'amato

Torre s. caterina

Torre del suvero seu Capo dell'Agliastro

Tab. 1. Le torri del litorale tirrenico.

\begin{tabular}{|c|c|c|}
\hline Tipologia & STIGLIOLA 1595 & BLANCH 1638 \\
\hline & & T. de Pentimele \\
\hline & T. de gallico & T. de Galico \\
\hline Cs & T. la Catona & T. de la Catona \\
\hline Q2 & T. flumaro de muro & \\
\hline Cs & T. lo peraino & T. del Piezzo \\
\hline Cs & T. S. Gregorio & T. del Cavallo \\
\hline Q2 & T. del Cavallo & \\
\hline Q2 & & \\
\hline Cs & T. de la bagnara & T. de la Bagnara \\
\hline Q1 & & \\
\hline Q2 & & \\
\hline Q1 & & \\
\hline Cs & T. de palma & T. de Palmi \\
\hline Q2 & & \\
\hline Cs & T. del C. petrenere & T. de las Piedras negras \\
\hline Cs & T. de gioia & T. de Jioja \\
\hline Q2 & & \\
\hline $\mathrm{Cs}$ & T. de rosarno & T. de Rosarno \\
\hline Q2 & & \\
\hline Q2 & & \\
\hline Cs & T. de Nicotera & T. de Nicotera \\
\hline Q1 & & \\
\hline $\mathrm{C}$ & T. de pizinaco & T. Santa Maria de Loreto \\
\hline Cs & T. C. Suvaro & T. cabo de Vaticano \\
\hline Q basam & T. della ruffa & T. de Rufa \\
\hline Cs & T. S. Domenico & T. de Santa Domenica \\
\hline Q1 & & \\
\hline $\mathrm{Cs}+\mathrm{Q} 1$ & T. del Capo Sanponio & T. de Zambrone \\
\hline Q1 & & \\
\hline Q2 & T. la Galera & T. Santa Arena \\
\hline Cs & T. de briatico & T. Imperial \\
\hline & T. la Rocchetta & \\
\hline Q2 & T. del Porto & T. Santo Nicola \\
\hline Cs & T. de bivona & \\
\hline & T. de praia & \\
\hline Cs & T. S. Venere & T. Santo Pedro \\
\hline & & T. del Pizo/del Bastion \\
\hline $\mathrm{Cs}+\mathrm{Q} 2$ & T. de agnone & T. Santa Venere \\
\hline $\mathrm{Cs}$ & T. dell'acque nere & T. de Media Playa \\
\hline Cs & T. de maida & T. dell'Amato \\
\hline Cs & $\mathrm{T} . \mathrm{S}^{\mathrm{ta}}$ Caterina & T. Santa Caterina \\
\hline Cs & T. C. Suvaro & T. del cabo del Suvaro \\
\hline
\end{tabular}


stesse strutture. Ho utilizzato invece il grassetto negli elenchi Stigliola e Blanch per le torri che non appaiono nel Codice o che corrispondono ad alcune che si suggeriva di costruire contrassegnando il luogo con una croce, come i quattro casi già citati. Tra quelle di cui il Codice propone la realizzazione troviamo confermate in Blanch due torri nel Cabo de las Columnas, che tuttavia non hanno assegnato un nome.
La torre n. 45 alla cala delli Porcelli, assente in Stigliola e in Blanch, era però stata appaltata nel 1598 al mastro Carolo Greco e nuovamente riappaltata nel 1600 e nel 1602, così come nel 1598 era aggiudicata al mastro Jo. Battista Fico di Cutro la torre n. 47 alla Cala di Posteriore, anch'essa poi riappaltata nel 1600 (Mafrici 2015, p. 50).
Torre Vedera

Torre Casamona

68-69 Torre San Fili +1 da costruire

70 Torre Ciamillarj, territorio di Castellovetere ROCCELLA DI CASTELVETERE Torre spina

72 Torre taburno, territorio della motta siderno
Tipologia

\section{STIGLIOLA 1595}

BLANCH 1638

\begin{tabular}{|c|c|c|}
\hline Q2 & $\begin{array}{l}\text { T. de Nieto } \\
\text { T. de Crepacore }\end{array}$ & \\
\hline Q2 & T. Capo de Colonne & Torre \\
\hline $\begin{array}{l}\text { Q2 } \\
\text { Q1 } \\
\text { Q1 }\end{array}$ & T. delle civette & Torre \\
\hline $\begin{array}{l}\text { Cs } \\
\text { Q1 }\end{array}$ & T. de manna & T. de Cabo Manna \\
\hline $\begin{array}{l}\text { Cs } \\
\text { Q2 } \\
\text { Q1 }\end{array}$ & T. C. Ricciuto & T. dello Rizzuto \\
\hline Q1 & T. delle Castelle & T. de li Castelle \\
\hline $\begin{array}{l}\text { Q1 } \\
\text { Q1 } \\
\text { Q2 }\end{array}$ & $\begin{array}{l}\text { T. de iacopani } \\
\text { T. lacozzia } \\
\text { T. de tacina }\end{array}$ & T. La Catenela \\
\hline Q basam & T. de cropani & T. de Crochia \\
\hline $\begin{array}{c}\text { Q basam + croce } \\
\text { Q2 } \\
\text { Q1 }\end{array}$ & T. de simari & T. de Simari \\
\hline $\mathrm{Cs}$ & $\begin{array}{l}\text { T. de Catanzaro } \\
\text { T. della rocca }\end{array}$ & $\begin{array}{l}\text { T. de Catanzaro } \\
\text { T. La Rocca }\end{array}$ \\
\hline $\begin{array}{l}\text { croce } \\
\text { Cs } \\
\text { Q1 } \\
\text { Q1 }\end{array}$ & T. de Stallati & T. Santa Maria de Vietri \\
\hline $\begin{array}{l}\mathrm{Cs}+\mathrm{Q} 1 \\
\mathrm{C} \text { basam }\end{array}$ & $\begin{array}{l}\text { T. di nisolizari } \\
\text { T. di taverna }\end{array}$ & \\
\hline $\mathrm{Cs}+\mathrm{Q} 1$ & T. de badulato & T. de Monte Manno \\
\hline $\begin{array}{c}\mathrm{C} \\
\mathrm{Cs}+\mathrm{Q} 2\end{array}$ & T. S. Antonio & $\begin{array}{l}\text { T. de S. Antonio } \\
\text { T. de Caminiti }\end{array}$ \\
\hline $\mathrm{Cs}$ & $\begin{array}{l}\text { T. de Castellone } \\
\text { T. della verità } \\
\text { T. de ficarazzo }\end{array}$ & T. del Castillon \\
\hline $\mathrm{Cs}$ & T. de vedra & T. de Edera \\
\hline $\mathrm{Cs}$ & T. de casamone & T. de Casa mona \\
\hline Cs + croce & T. S. Fili & T. de Santa File \\
\hline Cs & $\begin{array}{l}\text { T. de } \text { Cast }^{\circ} \text { vetere } \\
\text { T. della Rocella }\end{array}$ & $\begin{array}{l}\text { T. de Camilari } \\
\text { T. de la Rocha }\end{array}$ \\
\hline $\mathrm{Cs}$ & T. della spina & T. de la Spina \\
\hline $\mathrm{Cs}$ & T. de sidaro & T. de Tamuri \\
\hline
\end{tabular}

Tab. 2. Le torri del litorale tirrenico. 


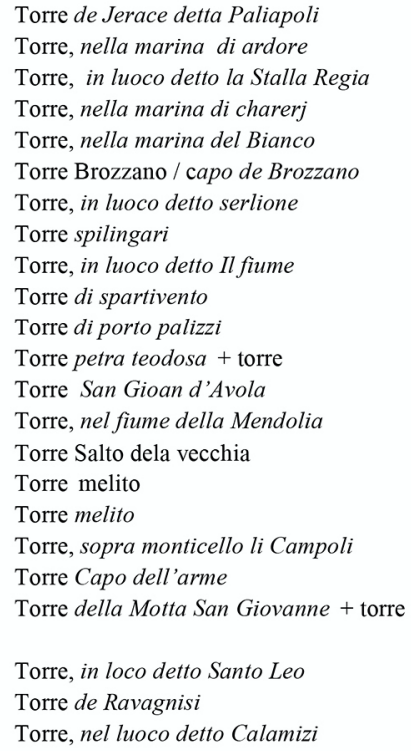

Tab. 3. Le torri del litorale tirrenico.

Credo che a quest'ultima possa essere riferita la torre de Iacopani dello Stigliola e La Catenela in Blanch: "La torre nomada La Catenela nel Territorio adonde se dize san Leonarde la qual no esta acabada y como los Padres jesuitas se han ofrezido de acabarla siendo ellos enteressados por del Territorio que alli tienen non digo otra cossa" (Valente 1964, p. 621).

La torre n. 36 da costruire nel Neto, presente in Stigliola, nel 1605 era ancora in costruzione e ne erano state realizzate 84 canne mentre in Blanch, come si è detto, sono indicate a Capo Colonna due torri senza alcun nome identificativo. La torre Mariello nel 1639 era ancora in costruzione (Mauro 1998, pp. 816, 818) ma risulta poi compiuta nel 1649 (Corrado, 2012, pp. 98-99). Ritengo poi che la torre n. 86 , da costruire nel fiume della Mendolia, sia stata realizzata perché presso questa fiumara in contrada S. Carlo nel territorio dell'attuale comune di Condofuri (RC) esiste una torre a pianta quadrangolare scarpata di ampie dimensioni, oggi priva della parte terminale con le caditoie in controscarpa.

\begin{tabular}{cll} 
Tipologia & STIGLIOLA 1595 & \multicolumn{1}{c}{ BLANCH 1638} \\
C & T. de Gerace & \\
croce & T. de pagliopoli & T. de Palla Poli \\
croce & & T. de la Mota Bovalina \\
Q basam & T. del bianco & T. de Passano \\
Q1 & & \\
Cs & T. del C. burzano & T. del Capo de Bruzano \\
Q2 & & \\
Cs & T. de Sperlinga & T. de Brancalione \\
Q1 & & \\
Cs & T. del C. Spartivento & T. de Spartivento \\
Q basam & T. de palizzi & T. de Puerto Palizzi \\
C + Q1 & T. S. Gio: Davila & T. de la Piedra \\
Cs & T. de bova & T. San Juan de Aila \\
Q2 & & \\
Cs & T. Salto la vecchia & T. del santo de la Vecha \\
Q basam & T. de melito & \\
Cs & T. de pintadattilo & T. de Melito \\
Q1 & T. del C. del arme & \\
Cs & T. la motta S. Giovanni & T. de Pelano \\
Cs + croce & T. del C. pellaro & \\
Q1 & & T. de Santa Agata \\
Cs & T. de Riggio & \\
Q1 & &
\end{tabular}

\section{Conclusioni}

In conclusione dal confronto effettuato si può affermare che nella prima metà del XVII secolo si era ben lontani dalla realizzazione della rete fitta e continua di capisaldi ideata già da un secolo e che questo progetto rimase un'utopia nonostante la continua attenzione, le ripetute proposte e le somme rilevanti impiegate.

L'importanza del Codice per il progetto di fortificazione con il dettaglio delle informazioni trasmesse, qui per brevità solo parzialmente commentate, conferma le spese rilevanti che la Corte avrebbe dovuto affrontare per la sua realizzazione, stimate alla fine del Cinquecento

in un totale di 80.375 ducati. Dimostra poi, nelle illustrazioni che lo arricchiscono, che fu utilizzato il metodo di rappresentazione delle opere fortificate introdotto per la prima volta nel 1592 da Carlo Gambacorta, marchese di Celenza Valfortore, che dichiarò di aver inventato il modo di "retrahere li luochi, che chiamo prospettiva, senz'altra misura" in occasione della Visita che 
effettuò nella Terra di Lavoro, Principato Citra e

Basilicata su incarico del vicerè conte di Miranda

(Mafrici 2015, pp. 46-48).

\section{Bibliography}

Bianchi, A.; Saeli, T. (2016). "Le fortificazioni costiere nella Calabria Ultra testimoniate dal Codice Romano Carratelli", in Verdiani, G. ed., Defensive Architecture of the Mediterranean. XV to XVIII Centuries, Didapress, Firenze, vol. III, pp. 29-32.

Conti, S.; Macrì, G.F. (2016). Le fortificazioni in Calabria Ultra all'epoca di Filippo II in un manoscritto inedito, in Verdiani, G. ed., Defensive Architecture of the Mediterranean. XV to XVIII Centuries, Didapress, Firenze, vol. III, pp. 411-418.

Corrado, M. (2012). Capo Colonna. Luci e ombre dal Medioevo al XX secolo, Città del Sole Edizioni, Reggio Calabria.

Corrado, M. (2016). “'Cartoline' dalla Calabria Ultra di fine Cinquecento. O no?”, in Verdiani, G. ed., Defensive Architecture of the Mediterranean. XV to XVIII Centuries, Didapress, Firenze, vol. III, pp. 63-70.

Galasso, G. (2005). "Il Regno di Napoli. Il Mezzogiorno spagnolo (1494-1622)”, in Galasso G. ed., Storia d'Italia, tom. XV, II, UTET Ed., Torino, pp. 301-1078.

García García, B.J. coord. (1993). Una relazione vicereale sul governo del Regno di Napoli agli inizi del Seicento, Bibliopolis, Napoli.

Hernando Sánchez, C.J. (2001). El reino de Nápoles en el imperio de Carlos V. La consolidatión de la conquista, Museo del Prado, Madrid.

Mafrici, M. (2009). "L'architetto e il territorio. La politica difensiva spagnola in Calabria", in Anselmi, A. coord., La Calabria del Viceregno spagnolo. Storia, arte, architettura, urbanística, Gangemi, Roma, pp. 741-757.

Mafrici, M. (2015). "Il Codice Romano Carratelli nel sistema difensivo del Regno di Napoli", in Martorano, F. coord., Progettare la difesa, rappresentare il territorio. Il Codice Romano Carratelli e la fortificazione nel Mediterraneo secoli XVI-XVII, Edizioni CSd'A, Reggio Calabria, pp. 43-66.

Martorano, F. (2015). "Progettare la difesa. Architetture, città, territorio nel Codice Romano Carratelli", in Martorano, F. coord., Progettare la difesa, rappresentare il territorio. Il Codice Romano Carratelli e la fortificazione nel Mediterraneo secoli XVI-XVII, Edizioni CSd'A, Reggio Calabria, pp. 67-102.

Mauro, A. (1998). Le fortificazioni nel regno di Napoli, Giannini Ed, Napoli.

Mazzamuto, A. (1986). Architettura e Stato nella Sicilia del '500, Atlante di Storia urbanistica siciliana, 8, Flaccovio Ed., Palermo.

Valente, G. (1964). Difesa e reclutamento di soldati in Calabria Ultra al tempo del vicario Giovan Tommaso Blanch. in La Calabria nel viceregno, $3^{\circ}$ Congresso storico calabrese, Deputazione Storia Patria per la Calabria, Napoli, pp. 607-680.

Valerio, V. (2015). “«Disegnare et ponere in pianta qualsivoglia sito del Regno», Il rilevamento del Regno di Napoli tra difesa militare e amministrazione civile", in Martorano, F. coord., Progettare la difesa, rappresentare il territorio. Il Codice Romano Carratelli e la fortificazione nel Mediterraneo secoli XVI-XVII, Edizioni CSd'A, Reggio Calabria, pp. 125-157. 